\title{
Influence of monolayer state on spectroscopy and photo-isomerization of an amphiphilic styryl-pyridinium dye on a solid substrate
}

\author{
Mariano L. Bossi, Andrey A. Turshatov, Sergei Yu. Zaitsev, Michael V. Alfimov, Dietmar Möbius \\ and Stefan W. Hell \\ Department of NanoBiophotonics, Max Planck Institute for Biophysical Chemistry, 37070 Göttingen, Germany; \\ Moscow State Academy of Veterinary Medicine and Biotechnology, Acad. Skryabina Str. 23, 109472 Moscow, \\ Russia; and Photochemistry Center, Russian Academy of Sciences, Novatorov Str. 72, 117334 Moscow, Russia.
}

\section{mbossi@gwdg.de and dmoebiu@gwdg.de}

\section{Fitting procedure of the polarized transmission spectra to Gaussian components}

Polarized absorption spectra of monolayers transferred at different surface pressures in the range $2.5 \mathrm{mN} / \mathrm{m}-25 \mathrm{mN} / \mathrm{m}$ were fitted to two or three Gaussian components. Figure A1 shows the fits obtained for two monolayers, one transferred from the liquid-expanded phase at $\pi=7 \mathrm{mN} / \mathrm{m}$, and the other transferred from the liquid-condensed phase at $\pi=20 \mathrm{mN} / \mathrm{m}$.
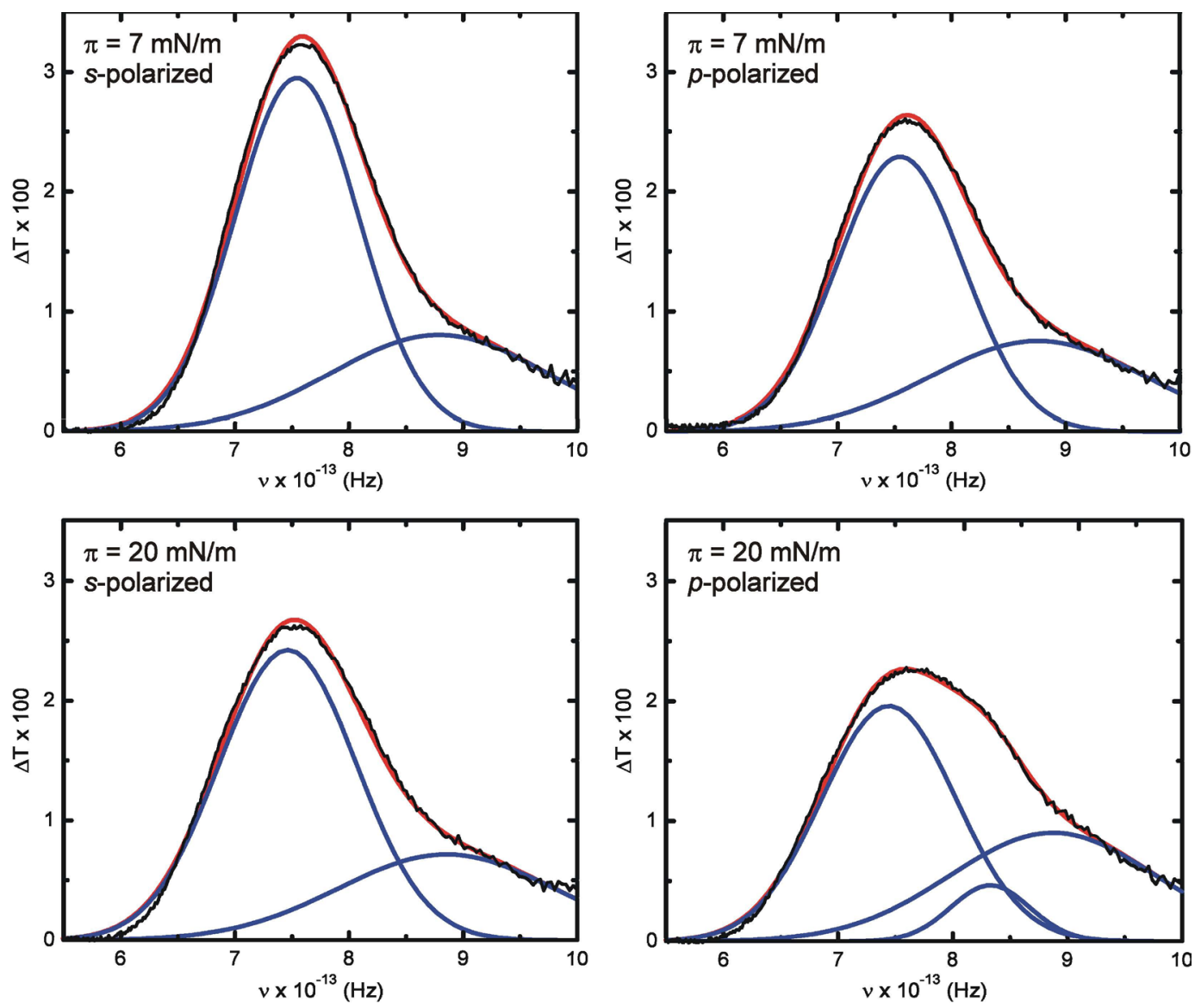

Figure A1. Polarized transmission spectra (left pots, $s$-polarized light; right plots, $p$-polarized light; angle of light incidence $45^{\circ}$ ) for monolayers transferred from the air-water interface to quartz plates at $\pi=7.0 \mathrm{mN} / \mathrm{m}$ (upper plots) and $\pi=20 \mathrm{mN} / \mathrm{m}$ (lower plots). Measured data is plotted in black lines, every Gaussian component is plotted with blue lines, and the red lines represent the total fitting function (sum of all Gaussian components). 
The s-polarized component could be fitted with two Gaussian functions for monolayers transferred at every surface pressure, regardless of the phase they were transferred from. The $p$ polarized component could also be fitted with two Gaussian functions for monolayers transferred from the liquid-expanded phase, but a third Gaussian component was necessary to fit the $p$ polarized spectra of monolayers transferred from the liquid-condensed phase. The parameters of the fits for all the monolayers transferred (three from the liquid-expanded phase $2.5 \mathrm{mN} / \mathrm{m}<\pi<$ $7 \mathrm{mN} / \mathrm{m}$; and three from the liquid condensed-phase $15 \mathrm{mN} / \mathrm{m}<\pi<25 \mathrm{mN} / \mathrm{m}$ ) are compiled in Table A1.

Table A1. Parameters of the fits of the polarized transmission spectra of monolayers transferred at different surface pressure $(\pi)$ to multiple Gaussian function (the band at the shortest wavelength is not included). The center $\left(\lambda_{\text {max }}\right)$, the full width at half maximum (FWHM), and the amplitude of the Gaussians (A) are shown for the $s$ - and $p$-polarized spectra, for the band centered at about $400 \mathrm{~nm}$ (blue band), and for the band centered at about $360 \mathrm{~nm}$ which is only present in the $p$ - polarized spectrum. The last two columns show the ratios of the amplitudes of the two bands observed in the $p$ - polarized component ( $\left.A_{p \cup v} / A_{p b l u e}\right)$, and the dichroic ratio of the blue band (Asblue / $\left.A_{p b l u e}\right)$

\begin{tabular}{|c|c|c|c|c|c|c|c|c|c|c|c|}
\hline \multirow{3}{*}{$\begin{array}{c}\pi \\
\mathrm{mN} / \mathrm{m}\end{array}$} & \multirow{2}{*}{\multicolumn{3}{|c|}{$\begin{array}{c}\text { s-polarized light } \\
\text { Blue band }\end{array}$}} & \multicolumn{6}{|c|}{ p-polarized light } & \multirow{3}{*}{$A_{\text {uV }}^{P} / A_{\text {blue }}^{P}$} & \multirow{3}{*}{$\begin{array}{l}\text { Dichroic ratio } \\
\text { (blue band) } \\
A_{\text {biue }}^{S} / A_{\text {biue }}^{D}\end{array}$} \\
\hline & & & & \multicolumn{3}{|c|}{ Blue band } & \multicolumn{3}{|c|}{ UV band } & & \\
\hline & $\begin{array}{l}\lambda_{\text {MAX }} \\
(\mathrm{nm})\end{array}$ & $\begin{array}{c}\text { FWHM } \\
\left(\mathrm{cm}^{-1}\right)\end{array}$ & $\begin{array}{l}A_{\text {blue }}^{s} \\
(\Delta T \%)\end{array}$ & $\begin{array}{l}\lambda_{\operatorname{MAX}} \\
(\mathrm{nm})\end{array}$ & $\begin{array}{c}F W H M \\
\left(\mathrm{~cm}^{-1}\right)\end{array}$ & $\begin{array}{l}A_{\text {blue }}^{D} \\
(\Delta T \%)\end{array}$ & $\begin{array}{l}\lambda_{\operatorname{MAX}} \\
(\mathrm{nm})\end{array}$ & $\begin{array}{c}F W H M \\
\left(\mathrm{~cm}^{-1}\right)\end{array}$ & $\begin{array}{c}A_{u V}^{p} \\
(\Delta T \%)\end{array}$ & & \\
\hline 2.5 & 398 & 4006 & 3.02 & 397 & 4042 & 2.29 & --- & --- & --- & --- & 1.32 \\
\hline 4.5 & 398 & 4068 & 2.75 & 397 & 4081 & 2.04 & --- & --- & --- & --- & 1.35 \\
\hline 7.0 & 400 & 4239 & 2.96 & 397 & 4356 & 2.29 & --- & --- & --- & --- & 1.29 \\
\hline 15 & 400 & 4551 & 2.21 & 402 & 4379 & 1.80 & 361 & 2991 & 0.35 & 0.19 & 1.23 \\
\hline 20 & 402 & 4721 & 2.42 & 403 & 4588 & 1.96 & 360 & 2700 & 0.47 & 0.24 & 1.24 \\
\hline 25 & 402 & 4755 & 2.43 & 405 & 4575 & 2.03 & 363 & 2999 & 0.65 & 0.32 & 1.20 \\
\hline
\end{tabular}

